\title{
Graphene-Based Biosensors for Diagnostics
}

\author{
A.T. Charlie Johnson \\ Department of Physics and Astronomy, University of Pennsylvania, Philadelphia USA \\ cjohnson@physics.upenn.edu
}

\begin{abstract}
:
We review recent results from our group in graphene-enabled biosensors for diagnostics and other applications. Scalable manufacturing approaches for graphene field effect transistors (GFETs) and graphene microelectrodes have been realized. GFET-based nucleic acid detectors based on complementary probes have a limit of detection of order $1 \mathrm{nM}$ for 20 mer and $1 \mathrm{aM}$ for 80 mer targets. The system is compatible with DNA engineering strategies including the use of hairpin probes and hybridization chain reaction as well as DNA aptamers for detection of small molecule targets. Biofunctionalized graphene microelectrodes are single-terminal devices that have been used as biosensors with pM sensitivity and specificity consistent with the chemical recognition element.
\end{abstract}

Key words: graphene, biosensor, field effect transistor, microelectrode, DNA.

\section{Introduction}

All-electronic biosensors based on biofunctionalized nano-devices offer a promising approach towards next-generation, label-free highly multiplexed diagnostics for use in the clinic, the home, or in a low-resource setting. Graphene is an ideal material for this application since it can be grown by large-area chemical vapor deposition (CVD) in a "layer" format [1] that is compatible with photolithographic processing [2]. Scalable fabrication procedures have been demonstrated for graphene with reproducible performance across devices and fabrication runs [3]. This gives graphene advantages at this time over one-dimensional nanomaterials (e.g., carbon nanotubes [4] and silicon nanowires [5]) or two-dimensional materials that are less well developed, e.g., the transition metal dichalcogenides [6,7].

Here we summarize recent results from our group. The focus is on two types of devices, three-terminal graphene field effect transistors (GFETs) and single terminal graphene microelectrodes [8]. For GFETs, transport through the graphene channel is modulated by electrostatic interactions with bound molecules [9], typically measured in the "dry state" [10], although measurements in fluid are also possible [11]. In contrast, for graphene microelectrodes the signal is spontaneous (i.e., zero-bias) Faradaic charge transport from fluid to the graphene, which is sensitive to $\mathrm{pH}$ [12], adsorbed species [13], and target binding [14].

\section{Results}

We have developed wafer-scale processes for scalable fabrication of arrays of 50-100 GFETs with excellent reproducibility, along with reliable functionalization with single-stranded DNA and DNA aptamers. The response of GFET DNA biosensors based on complementary probe DNA is defined as the shift in the GFET Dirac voltage, relative to the shift caused by exposure to targetfree buffer (Fig. 1a-b). The maximum (saturated) response, $A$, scales linearly with the length of the DNA target, consistent with electrostatic interactions due to deprotonation in residual water. The inferred dissociation constant $K_{A}$ and the associated limit of detection (LOD) decrease exponentially with target length, in agreement with earlier reports based on a quartz microbalance approach. Response curves are well described by the Sips model for DNA hybridization [15]. The LOD is $\sim 1$ aM for a $80-$ mer target.

The GFET system is compatible with more sophisticated approaches to DNA engineering. We have demonstrated the use of DNA aptamers for small molecule targets $[16,17]$. We have also used DNA hairpin probes and the hybridization chain reaction to enhance the sensitivity for 22-mers by $20,000 x$ compared to direct detection using a complementary probe [18]. 

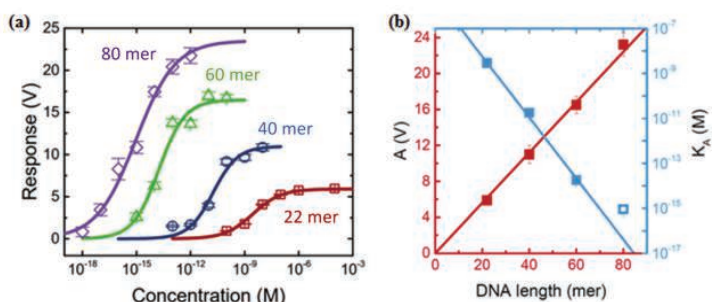

Fig. 1. (a) Relative Dirac voltage shift as a function of concentration for DNA targets of different lengths. Error bars (standard deviation of the mean) are approximately equal to the size of the plotted point. Solid curves are fits based on the Sips model. (b) Variation of maximum response $A$ (red data) and dissociation constant KA (blue data) with oligomer length. The lines are linear fits to the data.

Graphene microelectrodes are compatible with wafer-scale fabrication methods as well as implementation on flexible substrates [19]. For "bare" microelectrodes, the Faradaic current varies with ionic strength and $\mathrm{pH}$ in good agreement with predictions of theories of the electronic double layer. We recently demonstrated their use as a biosensor through functionalization with a computationally

\section{References}

[1] Z. Luo, Y. Lu, D. W. Singer, M. E. Berck, L. A. Somers, B. R. Goldsmith and A. T. C. Johnson, Chem. Mater. 23, 1441-1447 (2011).

[2] Z. Gao, H. Kang, C. H. Naylor, F. Streller, P. Ducos, M. D. Serrano, J. Ping, J. Zauberman, Rajesh, R. W. Carpick, Y.-J. Wang, Y. W. Park, Z. Luo, L. Ren and A. T. C. Johnson, ACS Appl. Mater. Interfaces 8 (41), 27546-27552 (2016).

[3] M. B. Lerner, D. Pan, Y. Gao, L. E. Locascio, K.-Y. Lee, J. Nokes, S. Afsahi, J. D. Lerner, A. Walker, P. G. Collins, K. Oegema, F. Barron and B. R. Goldsmith, Sensors and Actuators B: Chemical 239, 1261-1267 (2017).

[4] W. Yang, K. R. Ratinac, S. P. Ringer, P. Thordarson, J. J. Goodling and F. Braet, Angew. Chem., Int. Ed. 49, 2114-2138 (2010).

[5] Y. Cui, Q. Q. Wei, H. K. Park and C. M. Lieber, Science 293 (5533), 1289-1292 (2001).

[6] D. Sarkar, W. Liu, X. Xie, A. C. Anselmo, S. Mitragotri and K. Banerjee, ACS Nano 8 (4), 39924003 (2014).

[7] C. H. Naylor, N. J. Kybert, C. Schneier, J. Xi, G. Romero, J. G. Saven, R. Liu and A. T. C. Johnson, ACS Nano 10 (6), 6173-6179 (2016).

[8] J. Ping and A. T. C. Johnson, Appl. Phys. Lett. 109, 013103 (2016).

[9] M. B. Lerner, J. D'Souza, T. Pazina, J. Dailey, B. R. Goldsmith, M. K. Robinson and A. T. C. Johnson, ACS Nano 6, 5143-5149 (2012). redesigned, water-soluble variant of the human mu-opioid receptor (wsMOR). The Faradaic current (Fig. 2b) decreased systematically with the concentration of target analytes enkephalin and $\beta$-endorphin with a LOD at the pM level [14]. The system showed no response to the negative control oxytocin.
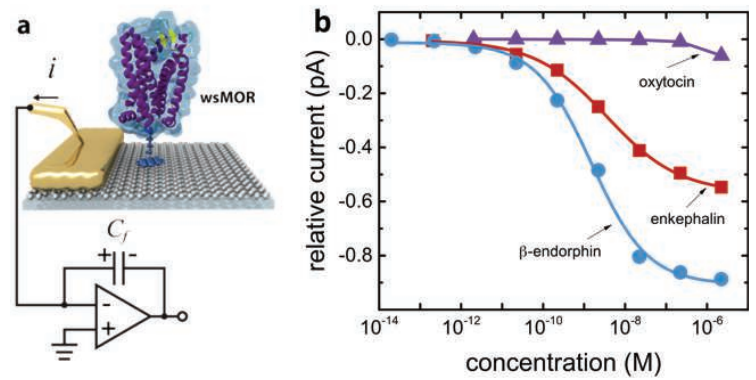

Fig. 2. (a) Schematic of the measurement system for charge transfer from a biosolution to a graphene microelectrode functionalized with water-soluble variant of the mu-opioid receptor (wsMOR). (b) Relative Faradaic current for enkephalin, $\beta$-endorphin and oxytocin as a negative control.

[10] J. Ping, R. Vishnubhotla, A. Vrudhula and A. T. C. Johnson, ACS Nano 10, 8700-8704 (2016).

[11] J. Ping, J. Xi, J. G. Saven, R. Liu and A. T. C. Johnson, Biosensors and Bioelectronics 15, 689-692 (2017).

[12] J. L. Ping, J. E. Blum, R. Vishnubhotla, A. Vrudhula, C. H. Naylor, Z. L. Gao, J. G. Saven and A. T. C. Johnson, Small 13 (30) (2017).

[13] J. Ping, K. W. Pulsipher, R. Vishnubhotla, J. A. Villegas, T. L. Hicks, S. Honig, J. G. Saven, I. J. Dmochowski and A. T. C. Johnson, Chemical Science 8, 5329-5334 (2017).

[14] J. Ping, R. Vishnubhotla, J. Xi, P. Ducos, J. G. Saven, R. Liu and A. T. C. Johnson, ACS Nano ASAP (2018).

[15] R. Sips, J. Chem. Phys. 16, 490-495 (1948).

[16] G. R. Wiedman, Y. Zhao, A. Mustaev, J. Ping, R. Vishnubhotla, A. T. C. Johnson and D. S. Perlin, mSphere 2, e00274-00217 (2017).

[17] R. Vishnubhotla, J. Ping, Z. Gao, A. Lee, O. Saouaf, A. Vrudhula and A. T. C. Johnson, AIP Advances 7, 115111 (2017).

[18] Z. Gao, H. Xia, J. Zauberman, M. Tomaiuolo, J. Ping, Q. Zhang, P. Ducos, S. Wang, H. Ye, X. Yang, F. Lubna, Z. Luo, L. Ren and A. T. C. Johnson, to appear in Nano Letters (2018).

[19] J. Ping, J. E. Blum, R. Vishnubhotla, A. Vrudhula, C. H. Naylor, Z. Gao, J. G. Saven and A. T. C. Johnson, Small (2017). 